\title{
REVISTA CHILENA DE DERECHO: TREINTA Y CINCO AÑOS AL SERVICIO DE LA COMUNIDAD JURÍDICA
}

\section{REVISTA CHILENA DE DERECHO: THIRTY FIVE YEARS SERVING LAW}

\author{
MARCELO BARRIENTOS ZAMORANO* \\ XIMENA SOTO TÉLLEZ** \\ MATÍAS ARÁNGUIZ VILLAGRÁN*** \\ FELIPE DUHALDE VERA ${ }^{* * * *}$
}

La Revista Chilena de Derecho (en adelante la Revista) cumple treinta y cinco años de vida. Es esta una publicación que representa hoy a la Facultad de Derecho de la Pontificia Universidad Católica de Chile en el mundo es su cara visible. Se encuentra inserta actualmente en índices internacionales, bibliotecas y hemerotecas, páginas y portales Web especializados en la investigación y el desarrollo del Derecho.

El sitial que hoy ocupa no ha sido sino el fruto del trabajo de personas que han pasado por su dirección y creación, la que hoy está a cargo de la profesora Ángela Vivanco Martínez y su Secretario de Redacción, el profesor Marcelo Barrientos Zamorano. Este trabajo tampoco sería posible sin el aporte generoso y entusiasta de todos los ayudantes y secretarias que a lo largo de este tiempo han contribuido a crear lo que hoy es la Revista Chilena de Derecho.

* Licenciado en Ciencias Jurídicas, Magíster en Derecho de la Empresa, Pontificia Universidad Católica de Chile, Doctor en Derecho con mención "Doctor Europeus", Universidad de Salamanca. Profesor de Derecho Civil de la Facultad de Derecho de la Pontificia Universidad Católica de Chile. Correo de contacto: mhbz@uc.cl

** Licenciada en Ciencias Jurídicas, Pontificia Universidad Católica de Chile. Correo de contacto: xssoto@uc.cl

*** Egresado de Derecho, Pontificia Universidad Católica de Chile. Correo de contacto: matiasrcv@gmail.com

**** Egresado de Derecho, Pontificia Universidad Católica de Chile. Correo de contacto: frduhald@uc.cl
En las líneas siguientes haremos un recorrido por estos treinta y cinco años, y con ello, hacemos un público reconocimiento a todas las mujeres y hombres que han contribuido en su creación y mantenimiento.

\section{HISTORIA DE LA REVISTA CHILENA DE DERECHO}

La Revista como tal fue fundada en el año 1974, apareciendo en febrero de ese año su primer número, luego de lo cual se ha seguido publicando hasta el día de hoy de forma ininterrumpida. Lo anterior en caso alguno supone que antes de 1974 no se realizaran publicaciones científicas en nuestra facultad. Las revistas especializadas en Derecho de la Facultad comenzaron hace setenta y tres años. En 1936 se empezaron a editar y publicar los "Anales Jurídicos-Sociales", publicación que tuvo una interrumpida divulgación por veintiséis años.

Entre 1972 y 1973 la Facultad publicó la revista "Estudios Jurídicos". En 1974 empezaron dos publicaciones nuevas: el "Boletín de Investigaciones" y la "Revista Chilena de Derecho”. El Boletín apareció por ocho años hasta 1982, donde pasó a ser parte de la Revista Chilena de Derecho, publicación que se mantiene con tres números al año, además de la publicación de eventuales ediciones especiales relacionadas con actividades de extensión.

Durante estos años la Revista ha contado con varios directores. El primero de ellos fue don Jaime Navarrete Barrueto (Q.E.P.D.), quien además de fundarla la dirigió hasta el año 1980. Luego le sucedió en el cargo don 
José Luis Cea Egaña, quien se encargó de consolidar el nombre de la Revista en el medio jurídico nacional, labor que realizó durante trece años hasta 1993. Desde 1994 a 1997 toma la dirección don Guillermo Bruna Contreras, período del que cabe destacar un hito importante, cual es, el que en 1995 se incorporaron los estudiantes de Derecho al trabajo de la Revista. Esto supuso incorporarlos como una más de las tantas formas en que nuestra Facultad educa a sus alumnos, enseñándoles a los que en ella trabajan la importancia de la investigación científica del Derecho y su divulgación.

Desde 1998 hasta 2007 estuvo a la cabeza de la Revista don Alejandro Vergara Blanco, quien dio gran importancia a la tarea de potenciar su carácter científico. Es mérito de Alejandro Vergara el inicio de la internacionalización e indexación de la Revista. La incorporación a índices internacionales y especialmente a SciELO fueron obras suyas. El índice SciElo es hoy pieza fundamental del vigor científico y dinamismo que ha demostrado la doctrina chilena en los últimos cinco años. Es este índice el que ha permitido a la Revista posicionarse a nivel internacional y nacional como una de las revistas más importantes en la ciencia jurídica de habla castellana.

Desde el año 2007 en adelante, y coincidiendo con el número 2 del volumen 34 , comienza su andadura el actual equipo de la Revista a cargo de la profesora Ángela Vivanco Martínez como su directora.

\section{OBJETIVOS DE LA REVISTA CHILENA DE DERECHO EN SU HISTORIA}

La Revista ha sido en sus treinta y cinco años una publicación especializada de Derecho, y que ha buscado abarcar todas sus especialidades dogmáticas y ciencias conexas, como la Filosofía y la Historia del Derecho.

Desde sus inicios la línea editorial de la publicación ha estado dirigida a lograr que los resultados de la investigación científica que se publican en sus páginas respondan a las necesidades de la comunidad jurídica nacional e internacional.
En ello se ha contado con el apoyo de la propia Pontificia Universidad Católica de Chile que ha entendido y fomentado la misión que le cabe a la Facultad, a través de la Revista, de difundir el pensamiento científico jurídico en la ciencia jurídica chilena y extranjera.

\section{ORGANIZACIÓN ACTUAL DE LA REVISTA CHILENA DE DERECHO}

A partir de 2007 en la Revista existen tres organismos importantes que reflejan el esfuerzo colectivo que cada número y volumen que representan: El Comité Editorial, el Comitée de Redacción y el Equipo de la Revista.

\section{COMITÉ EDITORIAL}

Es un órgano integrado por la Dirección de la Revista y un núcleo científico de académicos relevantes del mundo jurídico nacional y extranjero, todos ellos con trayectoria en el quehacer de la investigación y de las publicaciones jurídicas en diversas áreas del Derecho. Este comité se reúne anualmente para establecer las grandes políticas editoriales de la Revista, conocer su desarrollo y actividades, establecer pautas para intensificar su difusión y vinculación con diversas entidades y prestigiosos índices internacionales.

\section{COMITÉ DE REDACCIÓN}

Órgano integrado actualmente por el Sr. Decano Arturo Irarrázaval, el Vicedecano Sr. Roberto Guerrero, el Jefe de Carrera Sr. Carlos Frontaura, el Director de Investigación Sr. Marcelo Barrientos, el Secretario Ejecutivo del Programa de Doctorado Sr. Carlos Amunátegui, el Director de Doctorado y Magíster en Derecho Sr. Mario Correa, la Directora de la Revista Sra. Ángela Vivanco, más destacados miembros del claustro de profesores de la $\mathrm{Fa}$ cultad: Sr. Gonzalo Rojas, Sr. Patricio Carvajal, Sr. Enrique Alcalde, Sr. Juan José Romero y Sr. Álvaro Fernández.

Este comité se reúne periódicamente para informarse acerca de lo obrado por los diversos equipos de trabajo y discutir sobre los lineamientos a seguir, fijar los planes de acción y organizar los temas a tratar para darle un carácter general y científico a la Revista. 
EQUIPO DE LA REVISTA

Es el grupo de trabajo diario, conformado por la dirección y los ayudantes de marcaje y redacción. Este es un grupo de alumnos y ex alumnos formados en la actualidad por: Matías Aránguiz, Ximena Soto, Felipe Duhalde, María Gracia Toro, Carlos Pinto y Fernanda Pizarro. Forma también parte de este grupo la secretaria María Rosa Martínez y la directora de la Biblioteca de Derecho de la Universidad Sra. Evelyn Didier.

Todas estas personas y estamentos, más un cuerpo extenso de árbitros que desempeñan una labor anónima y gratuita encomiable, han posibilitado que la Revista se encuentre al día en las entregas de sus números y volúmenes desde mediados de 2007 hasta hoy.

Como puede apreciarse, la Revista Chilena de Derecho la formaron y mantuvieron por estos treinta y cinco años personas con un profundo sentido del Derecho, plenamente conscientes de la importancia de su estudio y divulgación para el mantenimiento del orden y la paz social. Es a este legado al que se debe el actual equipo que la forma y dirige, el que se reafirma en su historia y busca inspiración en ella para proyectar a la Facultad de Derecho, y a la propia Pontificia Universidad Católica de Chile, a los más importantes centros de estudio de Derecho en el mundo. 